\title{
Distinct Cortical Correlates of Autistic versus Antisocial Traits in a Longitudinal Sample of Typically Developing Youth
}

\author{
Gregory L. Wallace, ${ }^{1}$ Philip Shaw, ${ }^{2}$ Nancy Raitano Lee, ${ }^{2}$ Liv S. Clasen, ${ }^{2}$ Armin Raznahan, ${ }^{2}$ Rhoshel K. Lenroot, ${ }^{2}$ \\ Alex Martin, ${ }^{1}$ and Jay N. Giedd ${ }^{2}$ \\ ${ }^{1}$ Laboratory of Brain and Cognition and ${ }^{2}$ Child Psychiatry Branch, National Institute of Mental Health, NIH, Bethesda, Maryland 20892-1366
}

In humans, behaviors associated with autism and antisociality, disorders characterized by distinct social impairments, can be viewed as quantitative traits that range from frank impairment to normal variation, as found in the general population. Neuroimaging investigations of autism and antisociality demonstrate diagnostically specific aberrant cortical brain structure. However, little is known about structural brain correlates of social behavior in nonclinical populations. Therefore, we sought to determine whether autistic and antisocial traits exhibit dissociable cortical correlates and whether these associations are stable across development among typically developing youth. Three hundred twenty-three typically developing youth (age at first scan: mean $=10.63, \mathrm{SD}=3.71$ years) underwent anatomic magnetic resonance imaging ( $1-6$ scans each; total $=742$ scans), and provided ratings of autistic and antisocial traits. Higher autistic trait ratings were associated with thinner cortex most prominently in right superior temporal sulcus while higher antisocial trait ratings were associated with thinner cortex in primarily bilateral anterior prefrontal cortices. There was no interaction with age, indicating that these brain- behavior associations were stable across development. Using assessments of both subclinical autistic and subclinical antisocial traits within a large longitudinal sample of typically developing youth, we demonstrate dissociable neuroanatomic correlations that parallel those found in the frank clinical disorders of autism (e.g., superior temporal cortex) and antisociality (e.g., anterior prefrontal cortex). Moreover, these correlations appear to be established in early childhood and remain fixed into early adulthood. These results support the dimensional view of psychopathology and provide neural signatures that can serve as informative endophenotypes for future genetic studies.

\section{Introduction}

A fundamental question associated with the study of psychopathology is whether behavioral symptoms should be viewed dimensionally, wherein clinical disorders lie at the extreme of a continuous distribution of behaviors or traits (Rutter, 2003; Hudziak et al., 2007), or categorically. Recent research supports the concept that behaviors associated with both autism spectrum disorders (ASD; Constantino and Todd, 2003; Ronald et al., 2005; Skuse et al., 2005) and antisocial disorders (Frick et al., 2000; Murrie et al., 2007) can be viewed as such quantitative traits. Because these (social) behaviors are continuous and extend into the general population, we can seek to identify neural endophenotypes in large and relatively unconfounded (no comorbidities) subclinical samples.

Received Dec. 12, 2011; revised Feb. 1, 2012; accepted Feb. 16, 2012.

Author contributions: G.L.W. and J.N.G. designed research; G.L.W., N.R.L., L.S.C., and R.K.L. performed research; G.L.W. and P.S. analyzed data; G.L.W., P.S., A.R., A.M., and J.N.G. wrote the paper.

This research was supported by the Intramural Research Program of the NIH, National Institute of Mental Health. We thank the participants and their families who volunteered their time to contribute to this research.

The authors declare no competing financial interests.

Correspondence should be addressed to Gregory L. Wallace, Laboratory of Brain and Cognition, National Institute of Mental Health, 10 Center Drive, Room 4C104, MSC 1366, Bethesda, MD 20892-1366. E-mail: gregwallace@mail.nih.gov.

DOI:10.1523/JNEUROSCI.6214-11.2012

Copyright $\odot 2012$ the authors $\quad 0270-6474 / 12 / 324856-05 \$ 15.00 / 0$
Although ASD and antisocial disorders share impaired social functioning as a hallmark characteristic, they show distinctions in their genetic, cognitive, and neural profiles. Twin studies indicate that autistic and antisocial traits, though each highly heritable, have largely independent genetic underpinnings (Jones et al., 2009; Lundström et al., 2011). Cognitively, ASD is associated with impairments in reasoning about the mental states of others, whereas deficits in antisocial disorders are related to the processing of emotional stimuli, fear conditioning, and moral reasoning (Blair, 2008). These disorders also largely diverge in associated cortical anomalies. Three studies of cortical thickness converge in implicating thinner temporal and parietal cortices during adolescence/adulthood in high-functioning individuals with ASD compared with well matched controls (Hadjikhani et al., 2006; Wallace et al., 2010; Scheel et al., 2011) whereas antisocial disorders during adulthood (Yang et al., 2009) and disruptive behavior disorders during childhood (Fahim et al., 2011) are associated with thinner prefrontal and cingulate cortices.

Though the neuroanatomic atypicalities associated with these clinical syndromes are emerging, little is known of the neural correlates of subclinical social behavioral traits in typically developing (TD) children and adolescents. The few studies attempting to link autistic or antisocial traits with the brain in TD individuals have used functional neuroimaging methodologies with relatively small samples of adults (Rilling et al., 2007; Di Martino et 
al., 2009; Fullam et al., 2009; Buckholtz et al., 2010; Sheng et al., 2010). The literature on structural correlates is sparse: one study of adults found decreased white matter volume in the right posterior superior temporal sulcus with increasing autistic traits (von dem Hagen et al., 2011), and one study of children documented increasing gray matter concentration in left parahippocampal and left cingulate cortices with greater antisocial traits (Rijsdijsk et al., 2010). However, these studies were limited by relying on predefined regions of interest, volumetric methods, a single trait dimension (preventing examination of dissociation), and cross-sectional designs (preventing assessment of whether these cortical correlates are static/fixed or developmentally dynamic).

In a large cohort of TD youth, we examined the longitudinal, whole-brain cortical correlates of autistic and antisocial traits. Consistent with previous clinical studies documenting atypical superior temporal/temporoparietal cortices in ASD and aberrant prefrontal cortices in antisocial disorders, we predicted significant but largely orthogonal associations between cortical thickness and subclinical levels of autistic versus antisocial behaviors in TD youth.

\section{Materials and Methods}

Subjects. Three hundred twenty-three TD youth (176 males, 54\%; age range $=3.3-29.5$ years; Full Scale IQ estimate: mean $=111.92, \mathrm{SD}=$ 11.89; Hollingshead socioeconomic status score: mean $=43.26, \mathrm{SD}=$ 18.14) underwent MRI scanning. Subjects provided 1-6 scans each for a total of 742 structural MRI brain images; 323 subjects had at least one scan $(176$ males; age: mean $=10.63, \mathrm{SD}=3.71), 235$ had two scans $(133$ males; age: mean $=12.72, \mathrm{SD}=3.57), 110$ had three scans $(74$ males; age: mean $=14.38, \mathrm{SD}=3.26), 54$ had four scans $(43$ males; age: mean $=$ $16.91, \mathrm{SD}=3.42), 17$ had five scans ( 13 males; age: mean $=20.15, \mathrm{SD}=$ 5.37 ), and three had six scans (two males; age: mean $=18.73, \mathrm{SD}=5.37$ ). Subjects were part of a larger study of longitudinal typical brain development; thus, screening for the presence of learning, psychiatric, and neurologic disorders was the same as in previous studies (for details, see Giedd et al., 1999). Verbal or written assent was obtained from children and written consent from the parents or adult participants. The National Institute of Mental Health Institutional Review Board approved the protocol.

Neuroimaging. T1-weighted images with contiguous $1.5 \mathrm{~mm}$ axial slices and $2.0 \mathrm{~mm}$ coronal slices were obtained using 3-dimensional spoiled gradient recalled echo in the steady state on a 1.5T GE Signa scanner (GE Healthcare). Scans were registered into stereotaxic space, segmented (Zijdenbos et al., 2002) and the Constrained Laplacian Anatomic Segmentation Using Proximities surface extraction procedure was then used to generate surface meshes representing the white matter and gray matter interfaces (Kim et al., 2005). The root mean square thickness between corresponding nodes on the surface meshes was then calculated in native space (MacDonald et al., 2000). The cortical thickness measurements were aligned using surface registration to maximize thickness value correspondence between subjects in terms of gyral patterning (Lyttelton et al., 2007). A $30 \mathrm{~mm}$ surface blurring algorithm was used to reduce both the noise in the cortical thickness measurements and the effective number of statistical comparisons being performed (Chung et al., 2003).

Trait-based measures. All subjects also had a parent-completed rating (age completed: mean $=14.57, \mathrm{SD}=4.79$ ) from the Social Responsiveness Scale (SRS), which is used to assess primarily social traits associated with ASD across the full range of severity (i.e., from subclinical to clini$\mathrm{cal}$ ), distinguishes individuals with ASD from controls (Constantino et al., 2000), is highly correlated with a widely used diagnostic instrument, the Autism Diagnostic Interview (Constantino et al., 2003), and is developmentally stable (Constantino et al., 2009). The SRS is composed of 65 items (e.g., "has difficulty relating to peers" or "would rather be alone than with others"), each of which is rated as "not true," "sometimes true," "often true," or "almost always true."
The majority of subjects $(n=298)$ also had a score from the Antisocial Process Screening Device (APSD), a parent-completed (ages 7-13 years; $n=137$ ) or self-rating (ages $14+$ years; $n=161$ ) measure (age completed: mean $=14.66, \mathrm{SD}=4.68)$ that assesses temperamental, affective, and behavioral traits associated with antisocial behavior, is predictive of antisocial outcomes in children and adolescents (McMahon et al., 2010), and is temporally stable (Muñoz and Frick, 2007). The APSD is composed of 20 items (e.g., "lies easily and skillfully" or "acts without thinking of the consequences") each of which is rated as "not at all true," "sometimes true," or "definitely true." These APSD scores were associated with 704 of the 742 total scans in the SRS sample. Furthermore, this subset of 298 subjects providing APSD scores did not differ from the larger group of 323 individuals providing SRS data in terms of any demographic details ( 157 males, 53\%; age range $=3.6-29.5$ years; Full Scale IQ estimate: mean $=111.88, \mathrm{SD}=11.77$; Hollingshead socioeconomic status score: mean $=43.14, \mathrm{SD}=18.19$ ).

Total raw summary scores from both the SRS (mean $=22.39$, $\mathrm{SD}=$ 13.86 , range $=0-79$ ) and the APSD (mean $=9.36, \mathrm{SD}=4.72$, range $=$ $0-30$ ) were used in analyses here, as they are the most psychometrically sound indices based on factor analytic, reliability, and validity studies (Constantino et al., 2004; Poythress et al., 2006). Consistent with a screened, TD population, mean autistic trait (SRS) ratings and antisocial trait (APSD) ratings fell below scores expected in samples of individuals with ASD (mean SRS raw score $\geq 80$; Constantino et al., 2010) or conduct problems (mean APSD raw score >17; Vitacco et al., 2003).

Neither IQ nor parental socioeconomic status was significantly correlated with SRS and APSD scores ( $r$ values $<0.11$, $p$ values $>0.05$ ). Although age was modestly negatively correlated with SRS scores $(r=$ $-.20, p<0.001)$, it was not significantly associated with APSD scores $(r=0.03, p=0.58)$. Significant sex differences in APSD scores were found $(t=3.68, p<0.001)$ with males (mean $=10.29, \mathrm{SD}=4.63)$ rated as having more antisocial traits than females (mean $=8.32, \mathrm{SD}=4.62$ ). However, no such differences were noted in SRS scores $(t=0.11, p=$ $0.91)$. Finally, it should be noted that SRS and APSD scores were significantly positively correlated with one another $(r=0.39, p<0.001)$, though this correlation was not of sufficient magnitude to preclude examination of distinct neural correlates for each of these social trait measures.

Analysis. Mixed model regression was used here because, unlike other tools (e.g., repeated-measures ANOVA) used to examine developmental change, it allows the inclusion of multiple measurements per person, missing data, and irregular intervals between measurements (Pinheiro and Bates, 2000). Age effects on the relationship between cortical thickness and the APSD and SRS scores were tested first. We found no significant interaction between the age terms and the APSD and SRS scores throughout the cortex; thus, these interaction terms were dropped and the age terms were included as covariates. Additionally, sex, IQ, and parental socioeconomic status were included as covariates. In the final model, the $i$ th cortical thickness value of the $j$ th individual was modeled as

Thickness $_{\mathrm{ij}}=$ intercept $+\mathrm{d}_{\mathrm{j}}+\beta_{1}($ score $)+\beta \ldots \ldots$..(COVARIATES $)$

$$
+e_{\mathrm{ij}}
$$

where $d_{i}$ is a random effect modeling within-person dependence, the intercept and $\beta$ terms are fixed effects, and $e_{\mathrm{ij}}$ represents the residual error. The relationship between cortical thickness and the APSD/SRS score is given by the $\beta_{1}$ term. We adjusted for multiple comparisons by using the False Discovery Rate procedure (set at 0.05 ; Benjamini and Hochberg, 1995) and report FDR corrected significant clusters $>100$ vertices in extent.

\section{Results}

After accounting for contributions of age, sex, IQ, and socioeconomic status, we found that higher scores on the SRS, indicating greater autistic traits, were associated with thinner cortex in several regions (Fig. 1). The most prominent region centered on the posterior right superior/middle temporal gyrus (prominently along the superior temporal sulcus) extending into the angular gyrus $(t=4.0, p=0.0001)$. Association was also found in the 


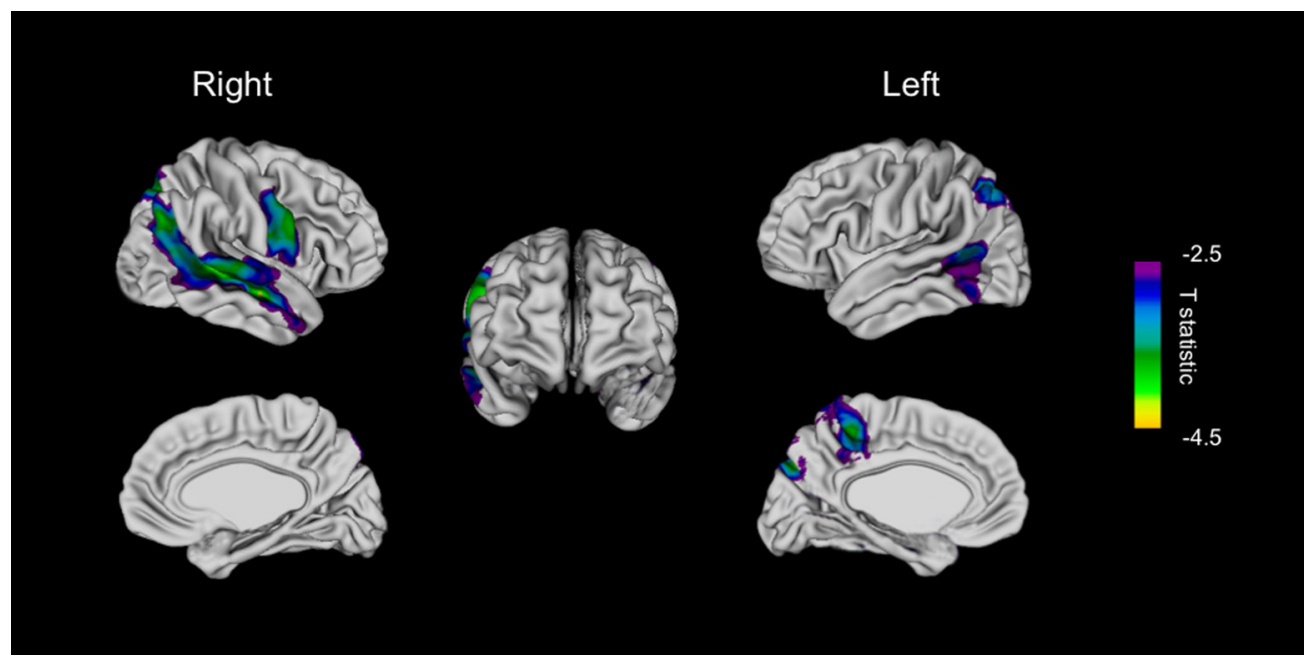

Figure 1. Correlations between cortical thinning and autistic traits after accounting for age, sex, socioeconomic status, IQ effects, and completing multiple-comparison corrections (using a false discovery rate of $\mathrm{q}<0.05)$.

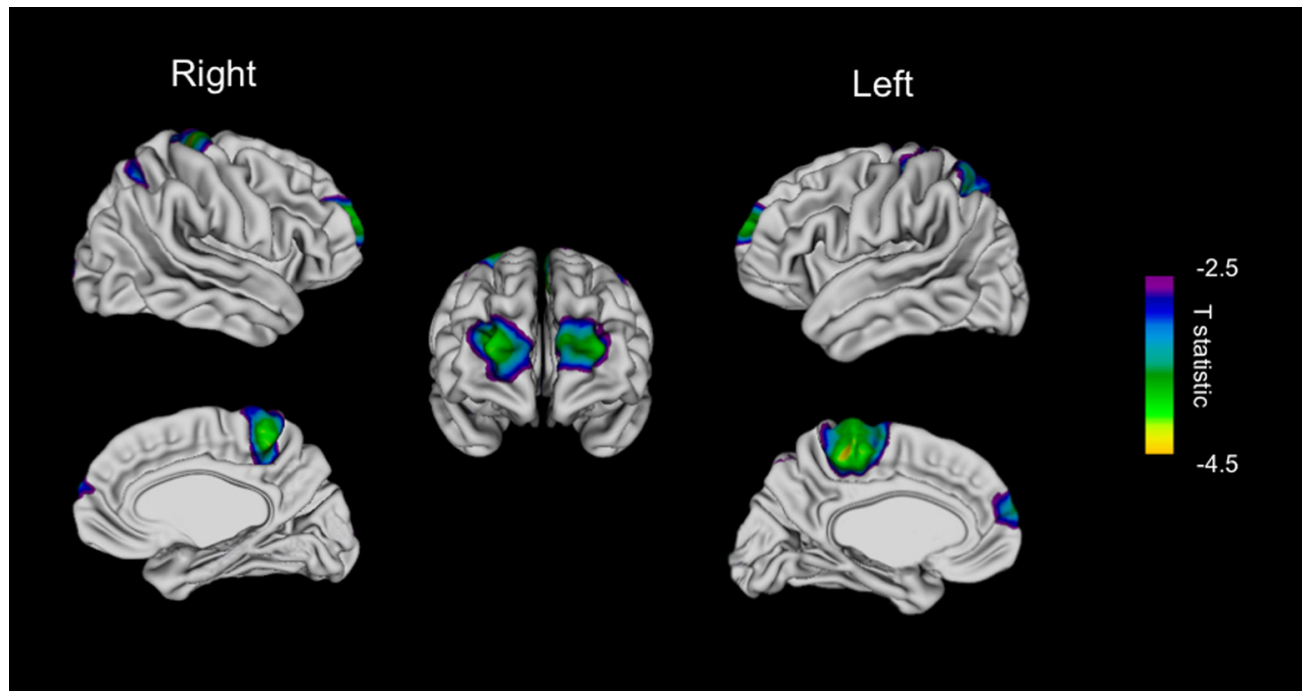

Figure 2. Correlations between cortical thinning and antisocial traits after accounting for age, sex, socioeconomic status, IQ effects, and completing multiple-comparison corrections (using a false discovery rate of $\mathrm{q}<0.05$ ).

left-sided posterior middle/superior temporal cortex $(t=3.8, p=0.0002)$, the right precentral gyrus $(t=3.8, p=$ 0.0002 ), and the left superior parietal lobule $(t=3.9, p=0.0002)$.

Increasing scores on the APSD, indicating more antisocial traits, were associated with thinning (also consistent across development) in different cortical regions, most prominently the anterior middle frontal gyri bilaterally $(t=3.6, p=0.0004)$, but also bilaterally in the medial portions of the precentral and postcentral/superior parietal cortices (for both the right and left, $t=2.7$, $p=0.008$; Fig. 2).

Notably, the cortical regions associated with autistic and antisocial traits were largely anatomically distinct with only one small region of overlap in portions of the left medial parietal cortex (Fig. 3).

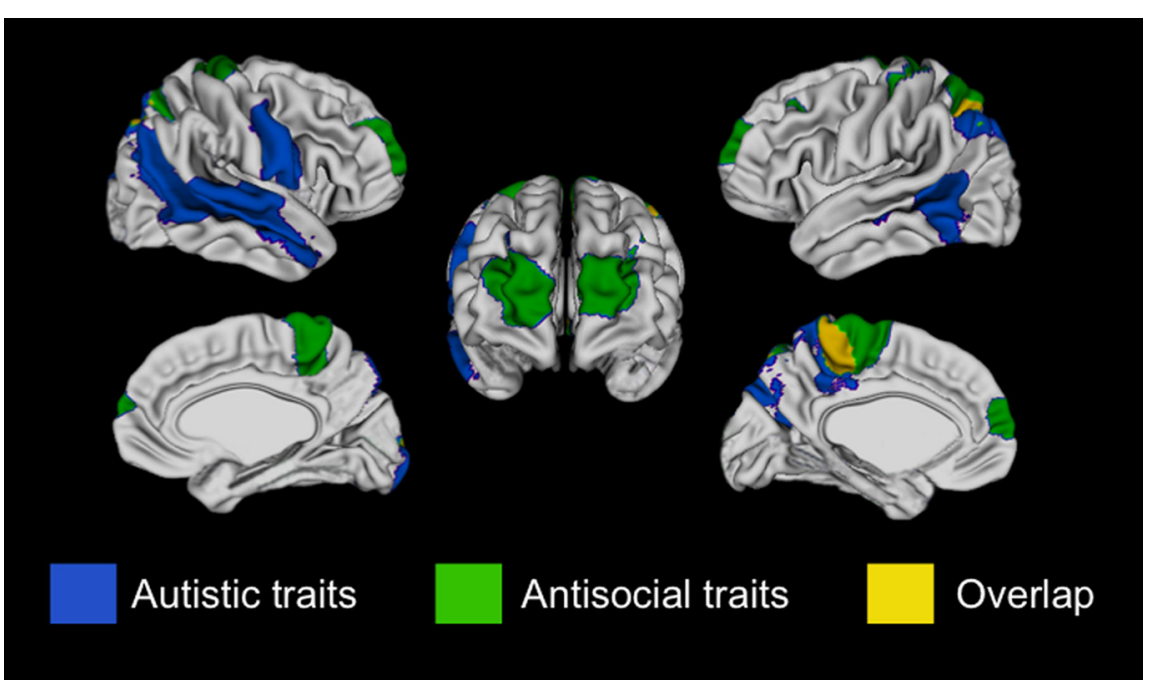

Figure 3. The spatial independence and overlap of autistic trait (in blue) and antisocial trait (in green) cortical correlates. 
These largely dissociable neural correlates were found despite the significant, albeit moderate, association between the two trait ratings in this sample.

\section{Discussion}

The present study provides the first neurobiological, particularly brain-based, evidence in support of a continuum underlying both ASD and antisocial disorders within a single large sample of typically developing individuals. Analysis of cortical thickness in a large cohort of TD youth revealed strikingly different cortical correlates for autistic and antisocial traits that remained stable across development. The superior temporal and temporoparietal regions, which correlated with autistic traits in our sample, overlap substantially with areas of cortical thinning in adolescents and adults with ASD (Hadjikhani et al., 2006; Wallace et al., 2010; Scheel et al., 2011) and with localized autistic trait/white matter volume correlations in neurotypical adults (von dem Hagen et al., 2011). These cortical regions have also been linked to social cognitive processing in healthy adults (Van Overwalle, 2009) and to functional anomalies in individuals with ASD during completion of "theory of mind" and other social cognitive tasks (Castelli et al., 2002; Pelphrey et al., 2011). Social cognition is a fundamental deficit among individuals with ASD, contributing to their struggles in forming and maintaining social interactions and relationships. By contrast, the cortical correlates of antisocial traits lie primarily in anterior prefrontal cortical regions where thinner cortex has been found in psychopathic adults (Yang et al., 2009) and in children with disruptive behavior disorders (Fahim et al., 2011) and where both functional and structural brain abnormalities have been associated with antisocial disorders (Yang and Raine, 2009). Anterior prefrontal cortices have also been linked with moral reasoning, among other functions, in neurotypical adults (Raine and Yang, 2006). Moral reasoning is a particular difficulty among individuals with antisocial behavior and may underlie their persistent violation of rules and social norms. Therefore, our results extend these cortical associations subclinically, bridging brain-behavior associations in the frank clinical disorders of ASD and antisociality with typical variations in the behaviors characterizing these disorders.

Importantly, we did not find a significant effect of age; the anatomically distinct cortical correlates of autistic and antisocial traits, previously shown to be temporally stable using the SRS (Constantino et al., 2009) and APSD (Muñoz and Frick, 2007), appeared early and held throughout childhood, adolescence, and young adulthood. Thus, fixed, distinct neuroanatomic signatures emerge, predominantly superior temporal/temporoparietal thinning for autistic traits and primarily anterior prefrontal cortical thinning for antisocial traits. Given the convergence of prior clinical studies and the subclinical associations demonstrated here, regionally specific cortical changes are candidate biomarkers for their related disorders. Therefore, the present brain-based findings extend prior studies demonstrating both largely separable genetic influences on autistic and antisocial traits (Jones et al., 2009; Lundström et al., 2011) and dissociable cognitive deficits in ASD and antisocial disorders (Rogers et al., 2006; Jones et al., 2010; Schwenck et al., 2011). Furthermore, these findings also inform the taxonomy of these disorders for DSM-5 (2013, in press) and beyond. We show not only dissociable cortical correlates for autistic versus antisocial traits, but we also do so subclinically, providing added validation for the dimensional examination of the behaviors associated with these disorders and trends toward taking a neuroscience-focused orientation to aid psychiatric classification (Insel et al., 2010).
There are limitations to consider. First, although communitybased, the sample was not epidemiological in nature. An epidemiological sample likely would have provided greater variance in social trait scores; however, using a well screened community sample allowed us to rule out variance due to other nuisance factors (e.g., comorbid psychopathology) known to affect brain structure. Also, the measure of antisocial traits (APSD) included both parent- and self-ratings, as determined by the age of the participant ( $<14$ years provided parent-ratings; $14+$ years provided self-ratings), raising the issue of potential rater effects. However, age did not affect correlations between antisocial traits and cortical thickness, and the results, as presented, included age as a covariate. Therefore, the potential influence of different APSD raters has been addressed in these analyses. Finally, the current sample comprised primarily school-age children and adolescents as well as young adults. Perhaps if the age range was extended lower, age effects moderating the relationship between cortical thickness and these social traits would have been detected.

Nevertheless, the present study, by being the first to examine correlations of both ASD and antisocial traits with cortical growth trajectories, implicates distinctive neural signatures for these social traits that extend subclinically into the general population. These dissociable neural correlates may serve as endophenotypes for ASD and antisocial disorders and thereby aid future genetic studies.

\section{References}

American Psychiatric Association (in press) Diagnostic and Statistical Manual of Mental Disorders, Ed 5, Washington, DC: American Psychiatric Association.

Benjamini Y, Hochberg Y (1995) Controlling the false discovery rate: a practical and powerful approach to multiple testing. J R Stat Soc B 57:289-300.

Blair RJ (2008) Fine cuts of empathy and the amygdala: dissociable deficits in psychopathy and autism. Q J Exp Psychol (Hove) 61:157-170.

Buckholtz JW, Treadway MT, Cowan RL, Woodward ND, Benning SD, Li R, Ansari MS, Baldwin RM, Schwartzman AN, Shelby ES, Smith CE, Cole D, Kessler RM, Zald DH (2010) Mesolimbic dopamine reward system hypersensitivity in individuals with psychopathic traits. Nat Neurosci 13:419-421.

Castelli F, Frith C, Happé F, Frith U (2002) Autism, Asperger syndrome and brain mechanisms for the attribution of mental states to animated shapes. Brain 125:1839-1849.

Chung MK, Worsley KJ, Robbins S, Paus T, Taylor J, Giedd JN, Rapoport JL, Evans AC (2003) Deformation-based surface morphometry applied to gray matter deformation. Neuroimage 18:198-213.

Constantino JN, Todd RD (2003) Autistic traits in the general population: a twin study. Arch Gen Psychiatry 60:524-530.

Constantino JN, Przybeck T, Friesen D, Todd RD (2000) Reciprocal social behavior in children with and without pervasive developmental disorders. J Dev Behav Pediatr 21:2-11.

Constantino JN, Davis SA, Todd RD, Schindler MK, Gross MM, Brophy SL, Metzger LM, Shoushtari CS, Splinter R, Reich W (2003) Validation of a brief quantitative measure of autistic traits: comparison of the Social Responsiveness Scale with the Autism Diagnostic Interview-Revised. J Autism Dev Disord 33:427-433.

Constantino JN, Gruber CP, Davis S, Hayes S, Passanante N, Przybeck T (2004) The factor structure of autistic traits. J Child Psychol Psychiatry 45:719-726.

Constantino JN, Abbacchi AM, Lavesser PD, Reed H, Givens L, Chiang L, Gray T, Gross M, Zhang Y, Todd RD (2009) Developmental course of autistic social impairment in males. Dev Psychopathol 21:127-138.

Constantino JN, Majmudar P, Bottini A, Arvin M, Virkud Y, Simons P, Spitznagel E (2010) Infant head growth in male siblings of children with and without autism spectrum disorders. J Neurodev Disord 2:39-46.

Di Martino A, Shehzad Z, Kelly C, Roy AK, Gee DG, Uddin LQ, Gotimer K, Klein DF, Castellanos FX, Milham MP (2009) Relationship between 
cingulo-insular functional connectivity and autistic traits in neurotypical adults. Am J Psychiatry 166:891-899.

Fahim C, He Y, Yoon U, Chen J, Evans A, Pérusse D (2011) Neuroanatomy of childhood disruptive behavior disorders. Aggress Behav 37:326-337.

Frick PJ, Bodin SD, Barry CT (2000) Psychopathic traits and conduct problems in community and clinic-referred samples of children: further development of the psychopathy screening device. Psychol Assess $12: 382-393$

Fullam RS, McKie S, Dolan MC (2009) Psychopathic traits and deception: functional magnetic resonance imaging study. Br J Psychiatry 194:229-235

Giedd JN, Blumenthal J, Jeffries NO, Castellanos FX, Liu H, Zijdenbos A, Paus T, Evans AC, Rapoport JL (1999) Brain development during childhood and adolescence: a longitudinal MRI study. Nat Neurosci 2:861-863.

Hadjikhani N, Joseph RM, Snyder J, Tager-Flusberg H (2006) Anatomical differences in the mirror neuron system and social cognition network in autism. Cereb Cortex 16:1276-1282.

Hudziak JJ, Achenbach TM, Althoff RR, Pine DS (2007) A dimensional approach to developmental psychopathology. Int J Methods Psychiatr Res $16: \mathrm{S} 16-\mathrm{S} 23$.

Insel T, Cuthbert B, Garvey M, Heinssen R, Pine DS, Quinn K, Sanislow C, Wang P (2010) Research domain criteria (RDoC): toward a new classification framework for research in mental disorders. Am J Psychiatry $167: 748-751$.

Jones AP, Larsson H, Ronald A, Rijsdijk F, Busfield P, McMillan A, Plomin R, Viding E (2009) Phenotypic and aetiological associations between psychopathic tendencies, autistic traits, and emotion attribution. Criminal Justice and Behav 36:1198-1212.

Jones AP, Happé FG, Gilbert F, Burnett S, Viding E (2010) Feeling, caring, knowing: different types of empathy deficit in boys with psychopathic tendencies and autism spectrum disorder. J Child Psychol Psychiatry 51:1188-1197.

Kim JS, Singh V, Lee JK, Lerch J, Ad-Dab'bagh Y, MacDonald D, Lee JM, Kim SI, Evans AC (2005) Automated 3-D extraction and evaluation of the inner and outer cortical surfaces using a Laplacian map and partial volume effect classification. Neuroimage 27:210-221.

Lundström S, Chang Z, Kerekes N, Gumpert CH, Råstam M, Gillberg C, Lichtenstein P, Anckarsäter H (2011) Autistic-like traits and their association with mental health problems in two nationwide twin cohorts of children and adults. Psychol Med 41:2423-2433.

Lyttelton O, Boucher M, Robbins S, Evans A (2007) An unbiased iterative group registration template for cortical surface analysis. Neuroimage 34:1535-1544.

MacDonald D, Kabani N, Avis D, Evans AC (2000) Automated 3-D extraction of inner and outer surfaces of cerebral cortex from MRI. Neuroimage 12:340-356.

McMahon RJ, Witkiewitz K, Kotler JS, Conduct Problems Prevention Research Group (2010) Predictive validity of callous-unemotional traits measured in early adolescence with respect to multiple antisocial outcomes. J Abnorm Psychol 119:752-763.

Muñoz LC, Frick PJ (2007) The reliability, stability, and predictive utility of the self-report version of the Antisocial Process Screening Device. Scand J Psychol 48:299-312.

Murrie DC, Marcus DK, Douglas KS, Lee Z, Salekin RT, Vincent G (2007) Youth with psychopathy features are not a discrete class: a taxometric analysis. J Child Psychol Psychiatry 48:714-723.

Pelphrey KA, Shultz S, Hudac CM, Vander Wyk BC (2011) Constraining heterogeneity: the social brain and its development in autism spectrum disorder. J Child Psychol Psychiatry 52:631-644.

Pinheiro JC, Bates DM (2000) Mixed-effects models in S and S-PLUS. New York: Springer.

Poythress NG, Douglas KS, Falkenbach D, Cruise K, Lee Z, Murrie DC, Vitacco M (2006) Internal consistency reliability of the self-report Antisocial Process Screening Device. Assessment 13:107-113.

Raine A, Yang Y (2006) Neural foundations to moral reasoning and antisocial behavior. Soc Cogn Affect Neurosci 1:203-213.

Rijsdijsk FV, Viding E, De Brito S, Forgiarini M, Mechelli A, Jones AP, McCrory E (2010) Heritable variations in gray matter concentration as a potential endophenotype for psychopathic traits. Arch Gen Psychiatry 67:406-413.

Rilling JK, Glenn AL, Jairam MR, Pagnoni G, Goldsmith DR, Elfenbein HA, Lilienfeld SO (2007) Neural correlates of social cooperation and noncooperation as a function of psychopathy. Biol Psychiatry 61:1260-1271.

Rogers J, Viding E, Blair RJ, Frith U, Happé F (2006) Autism spectrum disorder and psychopathy: shared cognitive underpinnings or double hit? Psychol Med 36:1789-1798.

Ronald A, Happé F, Plomin R (2005) The genetic relationship between individual differences in social and nonsocial behaviours characteristic of autism. Dev Sci 8:444-458.

Rutter M (2003) Categories, dimensions, and the mental health of children and adolescents. Ann NY Acad Sci 1008:11-21.

Scheel C, Rotarska-Jagiela A, Schilbach L, Lehnhardt FG, Krug B, Vogeley $\mathrm{K}$, Tepest $\mathrm{R}$ (2011) Imaging derived cortical thickness reduction in high-functioning autism: key regions and temporal slope. Neuroimage 58:391-400

Schwenck C, Mergenthaler J, Keller K, Zech J, Salehi S, Taurines R, Romanos M, Schecklmann M, Schneider W, Warnke A, Freitag CM (2011) Empathy in children with autism and conduct disorder: group-specific profiles and developmental aspects. Advance online publication. Retrieved January 13, 2012. J Child Psychol Psychiatry. doi: 10.1111/j.1469-7610.2011.02499.x.

Sheng T, Gheytanchi A, Aziz-Zadeh L (2010) Default network deactivations are correlated with psychopathic personality traits. PLoS One 5:e12611.

Skuse DH, Mandy WP, Scourfield J (2005) Measuring autistic traits: heritability, reliability and validity of the Social and Communication Disorders Checklist. Br J Psychiatry 187:568-572.

Van Overwalle F (2009) Social cognition and the brain: a meta-analysis. Hum Brain Mapp 30:829-858.

Vitacco MJ, Rogers R, Neumann CS (2003) The Antisocial Process Screening Device: an examination of its construct and criterion-related validity. Assessment 10:143-150.

von dem Hagen EA, Nummenmaa L, Yu R, Engell AD, Ewbank MP, Calder AJ (2011) Autism spectrum traits in the typical population predict structure and function in the superior temporal sulcus. Cereb Cortex 21:493-500.

Wallace GL, Dankner N, Kenworthy L, Giedd JN, Martin A (2010) Agerelated temporal and parietal cortical thinning in autism spectrum disorders. Brain 133:3745-3754.

Yang Y, Raine A (2009) Prefrontal structural and functional brain imaging findings in antisocial, violent, and psychopathic individuals: a metaanalysis. Psychiatry Res 174:81-88.

Yang Y, Raine A, Colletti P, Toga AW, Narr KL (2009) Abnormal temporal and prefrontal cortical gray matter thinning in psychopaths [letter]. Mol Psychiatry 14:561-562.

Zijdenbos AP, Forghani R, Evans AC (2002) Automatic "pipeline” analysis of 3-D MRI data for clinical trials: application to multiple sclerosis. IEEE Trans Med Imaging 21:1280-1291. 